DEPARTMENT OF THE INTERIOR

U.S. GEOLOGICAL SURVEY

\title{
Modern benthic foraminifer census data from box-cores collected on Northwind Ridge, Arctic Ocean during the PI-92-AR cruise of the U.S.C.G.C. Polar Star
}

Kevin M. Foley and Scott E. Ishman

U.S. Geological Survey, Reston, VA. 22092

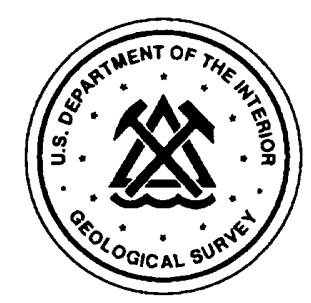

Open-File Report 93-515

This report is preliminary and has not been reviewed for conformity with U.S. Geological Survey Standards 
Benthic foraminifer census data from box-cores collected on Northwind Ridge, Arctic Ocean during the PI-92-AR cruise of the U.S.C.G.C. Polar Star

\author{
Kevin M. Foley and Scott E. Ishman \\ U.S. Geological Survey, Reston Va. 22092
}

\title{
INTRODUCTION
}

The data presented here are part of an interdisciplinary research effort to investigate the late Cenozoic climatic history of the Arctic Ocean. The study includes the integration of geophysical data with geochemical, sedimentologic, paleontologic and paleomagnetic data from marine cores in order to obtain a high resolution, comprehensive paleoclimatic and paleoceanographic record for the Arctic over the past $\approx 4.0$ My. Studies of the cores recovered in 1988 by the U.S. Coast Guard Cutter Polar Star showed that the Northwind Ridge contains an expanded sedimentary record that extends back into the early Pliocene (?). Additional geophysical data and marine sediment cores were obtained from the Northwind Ridge during the summer of 1992 (PI-92-AR) from the U.S. Coast Guard Cutter Polar Star. These cores included both piston and box cores. The foraminifer census data provided in this report represents the modern distribution of benthic foraminifers on the Northwind Ridge in 12 sediment surface samples taken from the PI-92-AR box cores (Figures 1, 2; Table 1).

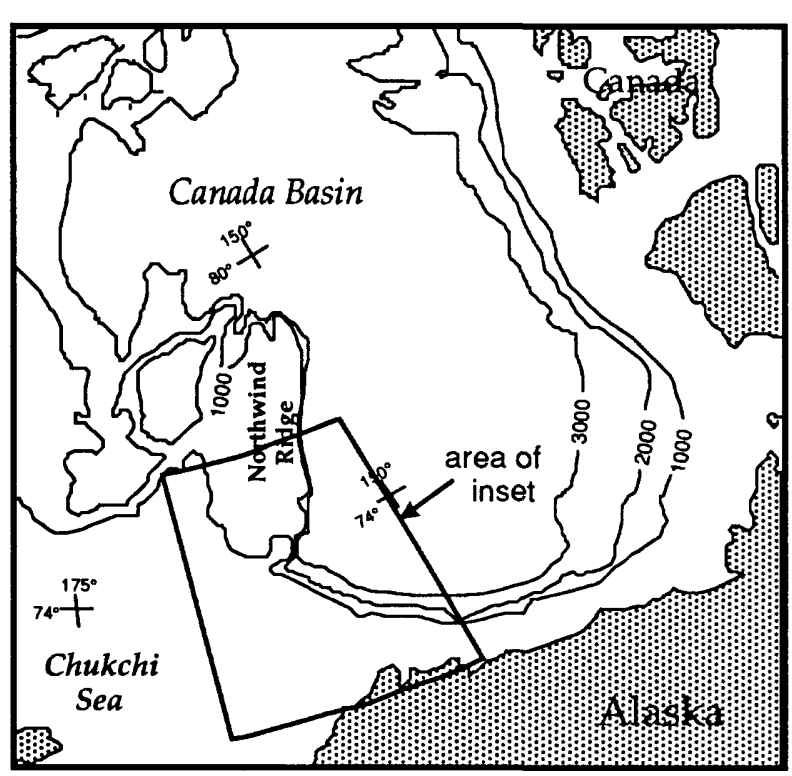

Figure 1 Map of central Arctic Ocean showing location of figure 1. (adapted from Gilbert and Clark, 1983)

\begin{tabular}{|cccc|}
\hline Core & Latitude & Longitude & Water Depth \\
\hline 1 & $72^{\circ} 24.2^{\prime} \mathrm{N}$ & $164^{\circ} 18.2^{\prime} \mathrm{W}$ & $49 \mathrm{~m}$ \\
2 & $72^{\circ} 23.8^{\prime} \mathrm{N}$ & $164^{\circ} 16.2^{\prime} \mathrm{W}$ & $48 \mathrm{~m}$ \\
3 & $73^{\circ} 41.6^{\prime} \mathrm{N}$ & $162^{\circ} 39.8^{\prime} \mathrm{W}$ & $201 \mathrm{~m}$ \\
4 & $74^{\circ} 00.0^{\prime} \mathrm{N}$ & $161^{\circ} 23.7^{\prime} \mathrm{W}$ & $447 \mathrm{~m}$ \\
5 & $74^{\circ} 30.6^{\prime} \mathrm{N}$ & $159^{\circ} 58.6^{\prime} \mathrm{W}$ & $585 \mathrm{~m}$ \\
7 & $74^{\circ} 49.2^{\prime} \mathrm{N}$ & $157^{\circ} 33.5^{\prime} \mathrm{W}$ & $1,055 \mathrm{~m}$ \\
8 & $74^{\circ} 49.4^{\prime} \mathrm{N}$ & $157^{\circ} 25.5^{\prime} \mathrm{W}$ & $1,402 \mathrm{~m}$ \\
9 & $74^{\circ} 49.5^{\prime} \mathrm{N}$ & $157^{\circ} 12.3^{\prime} \mathrm{W}$ & $2,120 \mathrm{~m}$ \\
12 & $74^{\circ} 49.5^{\prime} \mathrm{N}$ & $156^{\circ} 52.4^{\prime} \mathrm{W}$ & $3,811 \mathrm{~m}$ \\
15 & $75^{\circ} 44.1^{\prime} \mathrm{N}$ & $160^{\circ} 51.6^{\prime} \mathrm{W}$ & $2,135 \mathrm{~m}$ \\
16 & $75^{\circ} 43.7^{\prime} \mathrm{N}$ & $160^{\circ} 05.5^{\prime} \mathrm{W}$ & $1,388 \mathrm{~m}$ \\
17 & $76^{\circ} 05.2^{\prime} \mathrm{N}$ & $164^{\circ} 50.1^{\prime} \mathrm{W}$ & $402 \mathrm{~m}$ \\
\hline
\end{tabular}

Table 1 Latitude, longitude, and water depth for PI-92-AR box-cores shown in Figure 1.

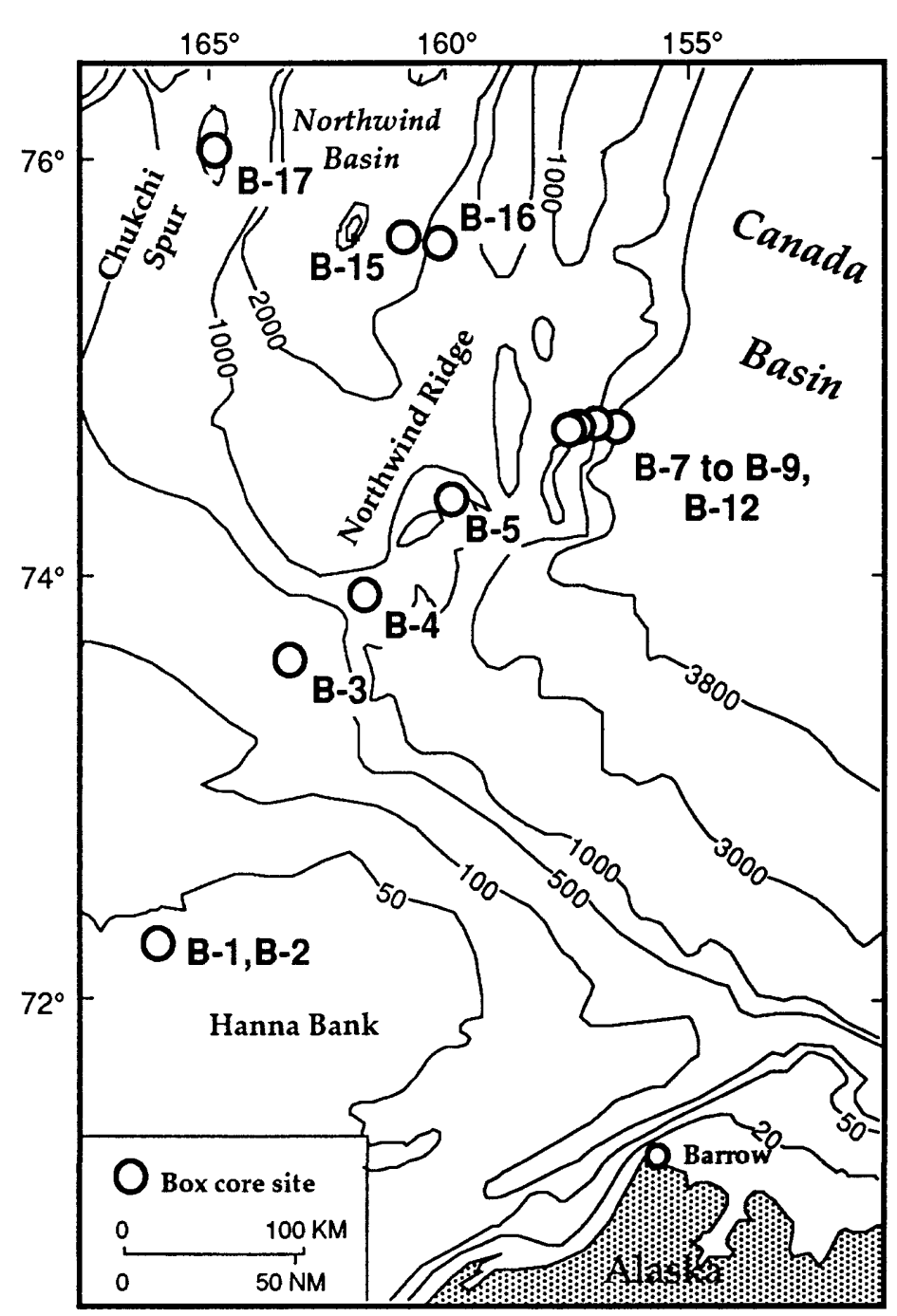

Figure 2 Map of Northwind Ridge showing the location of cores PI-92-AR box-cores. (adapted from Mullen and Grantz, 1991) 


\section{METHODS}

\section{Sampling}

Samples of approximately $50 \mathrm{cc}$ 's were taken from the top 2 centimeters of sediment within 24 hours of the collection of the core.

\section{Processing}

The samples used in this study were processed at the U.S. Geological Survey, Reston. Sediment samples were dried at $\leq 50^{\circ} \mathrm{C}$, weighed, and re-suspended in $200 \mathrm{ml}$ of dilute calgon solution. The samples were agitated for a period of one hour to assist in disaggregation, then wet sieved at $63 \mu \mathrm{m}$. The $\geq 63 \mu \mathrm{m}$ residue was dried at $\leq 50^{\circ} \mathrm{C}$.

\section{Counting}

All benthic foraminifers were tabulated in samples from cores B1, B2, B3, B5 and $\mathrm{B} 12$. Benthic foraminifers from cores $\mathrm{B} 4$, $\mathrm{B} 7, \mathrm{~B} 8, \mathrm{~B} 9, \mathrm{~B} 15, \mathrm{~B} 16$ and $\mathrm{B} 17$ were tabulated from representative subsamples obtained with a microsplitter. The sample or split to be examined was dry-sieved to the following size fractions; $\geq 63 \mu \mathrm{m}$ to $106 \mu \mathrm{m}$, $\geq 106 \mu \mathrm{m}$ to $125 \mu \mathrm{m}, \geq 125 \mu \mathrm{m}$ to $150 \mu \mathrm{m}$, and $\geq 150 \mu \mathrm{m}$. All benthic foraminifers were picked from each size fraction, sorted by species and glued to a standard 60-square micropaleontological slide. Data for each sample were tallied by species and size fraction.

\section{BACKGROUND}

Analyses of core-top samples and other surface sediment samples from the Arctic Ocean (Green, 1960; Lagoe, 1977, 1979; Polyak, 1990; and Scott and Vilks, 1991) provide information on the distribution of benthic foraminifers in parts of the Arctic Ocean. These data, in addition to the data presented in this report will add to our knowledge of benthic foraminifers in the Arctic Ocean. Associations made between intermediate and deep water masses and benthic foraminifer distributions provide the basis for paleoceanographic interpretations.

\section{BENTHIC FORAMINIFER TAXONOMY}

Benthic foraminifers from the PI-92-AR box cores were classified in accordance with Loeblich and Tappan, 1988. Where possible, the identifications are based on comparisons made with previously published Arctic taxa. A number of taxa could not be identified to species level and are referred herein as "sp." or "spp.". The benthic foraminifer taxa and their abundance in the PI-92-AR box core top samples are listed in Table 2.

\section{ACKNOWLEDGMENTS}

We would like to thank Suzy Greene and Anne Rogers for technical assistance.

\section{REFERENCES}

Buzas, M.A., 1972. Patterns of species diversity and their explanation. Taxon, 21 (2/3) May: pp. 275-286.

Gilbert, M. W. and Clark, D. L., 1983. Central Arctic Ocean paleoceanographic interpretations based on Late Cenozoic calcareous dinoflagellates. Marine Micropaleontology. v.7, p. 385-401.

Green, K.E., 1960. Ecology of some Arctic foraminifera. Micropaleontology, vol. 6 , no. 1 , pp. 57-78.

Lagoe, M.B., 1977. Recent Benthic Foraminifera from the Central Arctic Ocean. Journal of Foraminiferal Research, v. 7, no. 2, p. 106-129.

1979. Recent benthonic foraminiferal biofacies in the Arctic Ocean. Micropaleontology, vol. 25, pp. 214-224

Loeblich, A.R., Jr. and Tappan, H., 1988. Foraminiferal Genera and Their Classification, Volume 1, 970 pp., Van Nostrand Reinhold, New York.

Mullen, M. W., Grantz, A., 1991. Bathymetric Map of Southern Northwind Ridge and Vicinity, Arctic 
Ocean: USGS Open-file report 91136 , scale 1:250,000.

Polyak, L. V., 1990. General trends of benthic foraminiferal distribution in the Arctic Ocean. In: V. M. Kotlyakov and V.E. Sokolov (Editors), Arctic Research: Advances and Prospects: proceedings of the Conference of Arctic and Nordic
Countries on Coordination of Research in the Arctic. Nauka, Leningrad, p. 447.

Scott, D.B., Vilks, G., 1991. Benthonic foraminifera in the surface sediments of the deep-sea Arctic Ocean. Journal of Foraminiferal Research, v. 21, no. 1, p. 20-38.

Table 2 Occurrences of benthic foraminifers are tabulated by species and by size fraction from which specimens were extracted. Species total = the sum of the number of each taxon in all size fractions. Size fraction total $=$ the sum of the number of all taxa in one size fraction. Percent of total count = (number of benthic foraminifers found in size fraction) / (number of benthic foraminifers found in sample) X 100. S-W index is the Shannon Wiener index of species diversity ( see Buzas, 1972). 


\begin{tabular}{|c|c|c|c|c|c|c|c|c|c|c|}
\hline \multirow[b]{2}{*}{ TAXA size $(\mu \mathrm{m})$} & \multicolumn{5}{|c|}{$\begin{array}{l}\text { PI-92-AR-B2, water depth } 48 \mathrm{M} \\
\text { entire sample examined Species }\end{array}$} & \multicolumn{5}{|c|}{$\begin{array}{l}\text { PI-92-AR-B1, water depth } 49 \mathrm{M} \\
\text { entire sample examined Species }\end{array}$} \\
\hline & 63-106 & $106-125$ & $125-150$ & $>150$ & total & 63-106 & $106-125$ & $125-150$ & $>150$ & total \\
\hline Adercotryma glomerata & 0 & 0 & 0 & 0 & 0 & 0 & 0 & 0 & 0 & 0 \\
\hline Alveophragmium crassimargo & 0 & 0 & 0 & 0 & 0 & 0 & 0 & 0 & 0 & 0 \\
\hline Ammodiscus catinus & 0 & 0 & 0 & 0 & 0 & 0 & 0 & 0 & 0 & 0 \\
\hline Ammotium cassis & 0 & 0 & 7 & 16 & 23 & 3 & 3 & 0 & 5 & 11 \\
\hline Astrononion gallowayi & 0 & 0 & 0 & 1 & 1 & 0 & 0 & 0 & 0 & 0 \\
\hline Bolivina arctica & 0 & 0 & 0 & 0 & 0 & 1 & 0 & 0 & 0 & 1 \\
\hline Bolivina pseudopunctata & 0 & 0 & 0 & 0 & 0 & 0 & 0 & 0 & 0 & 0 \\
\hline Buccella frigida & 8 & 10 & 16 & 0 & 34 & 0 & 0 & 1 & 0 & 1 \\
\hline Bulimina aculeata & 0 & 0 & 0 & 0 & 0 & 0 & 0 & 0 & 0 & 0 \\
\hline Cassidulina norcrossi & 0 & 0 & 0 & 0 & 0 & 0 & 0 & 0 & 0 & 0 \\
\hline Cassidulina teretis & 0 & 0 & 0 & 0 & 0 & 0 & 0 & 0 & 0 & 0 \\
\hline Chilostomella elongata & 0 & 0 & 0 & 0 & 0 & 0 & 0 & 0 & 0 & 0 \\
\hline Cibicides lobatulus & 0 & 0 & 0 & 0 & 0 & 0 & 0 & 0 & 0 & 0 \\
\hline Cribroelphidium clavatum & 0 & 0 & 0 & 0 & 0 & 0 & 0 & 0 & 0 & 0 \\
\hline Cribroelphidium excavatum & 26 & 22 & 75 & 33 & 156 & 10 & 13 & 31 & 27 & 81 \\
\hline Cribrostomoides sp. & 0 & 0 & 0 & 1 & 1 & 0 & 0 & 0 & 0 & 0 \\
\hline Cystammina argentea & 0 & 0 & 0 & 0 & 0 & 0 & 0 & 0 & 0 & 0 \\
\hline Dentalina pauperata & 0 & 0 & 0 & 0 & 0 & 0 & 0 & 0 & 0 & 0 \\
\hline Discorbis squamata & 0 & 0 & 0 & 0 & 0 & 0 & 0 & 0 & 0 & 0 \\
\hline Eggerella advena & 1 & 3 & 5 & 15 & 24 & 2 & 1 & 9 & 0 & 12 \\
\hline Eponides tumidulus & 0 & 0 & 0 & 0 & 0 & 0 & 0 & 0 & 0 & 0 \\
\hline Fontbotia wuellerstorfi & 0 & 0 & 0 & 0 & 0 & 0 & 0 & 0 & 0 & 0 \\
\hline Fursenkoina fusiformis & 87 & 13 & 38 & 0 & 138 & 11 & 4 & 1 & 0 & 16 \\
\hline Glandulina laevigata & 0 & 0 & 1 & 0 & 1 & 0 & 0 & 0 & 2 & 2 \\
\hline Globocassidulina subglobosa & 2 & 4 & 3 & 1 & 10 & 0 & 0 & 0 & 1 & 1 \\
\hline Glomospira gordialis & 0 & 0 & 0 & 0 & 0 & 0 & 0 & 0 & 0 & 0 \\
\hline Islandiella helenae & 0 & 0 & 0 & 0 & 0 & 0 & 0 & 0 & 0 & 0 \\
\hline Melonis barleeanum & 0 & 0 & 0 & 0 & 0 & 0 & 0 & 0 & 0 & 0 \\
\hline Nodosariids & 0 & 0 & 0 & 0 & 0 & 0 & 1 & 0 & 0 & 1 \\
\hline Nonionellina labradorica & 0 & 1 & 2 & 0 & 3 & 0 & 0 & 0 & 1 & 1 \\
\hline Oridorsalis tener & 0 & 0 & 0 & 0 & 0 & 0 & 0 & 0 & 0 & 0 \\
\hline Oridorsalis umbonatus & 0 & 0 & 0 & 0 & 0 & 0 & 0 & 0 & 0 & 0 \\
\hline Portatrochammina bipolaris & 10 & 3 & 3 & 0 & 16 & 13 & 2 & 0 & 0 & 15 \\
\hline Pullenia quinqueloba & 0 & 0 & 0 & 0 & 0 & 0 & 0 & 0 & 0 & 0 \\
\hline Quinqueloculina akneriana & 0 & 0 & 0 & 0 & 0 & 0 & 0 & 0 & 1 & 1 \\
\hline Quinqueloculina arctica & 0 & 0 & 0 & 0 & 0 & 0 & 0 & 0 & 0 & 0 \\
\hline Recurvoides scitulus & 0 & 0 & 0 & 0 & 0 & 0 & 0 & 0 & 0 & 0 \\
\hline Reophax scorpiurus & 0 & 0 & 0 & 0 & 0 & 0 & 0 & 0 & 2 & 2 \\
\hline Reophax spp. & 0 & 0 & 0 & 0 & 0 & 0 & 0 & 0 & 0 & 0 \\
\hline Robertinoides charlottensis & 0 & 0 & 0 & 0 & 0 & 0 & 0 & 0 & 0 & 0 \\
\hline Rosalina globularis & 0 & 0 & 0 & 0 & 0 & 0 & 0 & 0 & 0 & 0 \\
\hline Saccammina difflugiformis & 0 & 0 & 0 & 1 & 1 & 0 & 0 & 0 & 0 & 0 \\
\hline Spiroplectammina biformis & 425 & 335 & 58 & 5 & 823 & 47 & 104 & 7 & 0 & 158 \\
\hline Stainforthia concava & 0 & 0 & 0 & 0 & 0 & 0 & 0 & 0 & 0 & 0 \\
\hline Stetsonia horvathi & 1 & 3 & 0 & 0 & 4 & 7 & 11 & 2 & 2 & 22 \\
\hline Textularia kattagatensis & 284 & 1 & 2 & 0 & 287 & 28 & 0 & 0 & 0 & 28 \\
\hline Textularia torquata & 368 & 34 & 3 & 0 & 405 & 87 & 4 & 1 & 0 & 92 \\
\hline Triloculina frigida & 0 & 0 & 0 & 0 & 0 & 0 & 0 & 0 & 0 & 0 \\
\hline Trochammina pseudoinflata & 0 & 0 & 0 & 0 & 0 & 0 & 0 & 0 & 0 & 0 \\
\hline Trochammina subglobigeriniformis & 0 & 0 & 0 & 0 & 0 & 0 & 0 & 0 & 0 & 0 \\
\hline Valvulineria arctica & 1 & 1 & 0 & 0 & 2 & 0 & 0 & 0 & 0 & 0 \\
\hline Verneuilina minuta & 0 & 0 & 0 & 0 & 0 & 0 & 0 & 0 & 0 & 0 \\
\hline Size fraction total & 1213 & 430 & 213 & 73 & 1929 & 209 & 143 & 52 & 41 & 445 \\
\hline Percent of Total Count & 62.88 & 22.29 & 11.04 & 3.78 & 100.0 & 46.97 & 32.13 & 11.69 & 9.21 & 100.0 \\
\hline S-W Index & 0.67 & 0.44 & 0.69 & 0.54 & 0.86 & 0.73 & 0.46 & 0.54 & 0.54 & 0.83 \\
\hline
\end{tabular}




\begin{tabular}{|c|c|c|c|c|c|c|c|c|c|c|}
\hline \multirow[t]{2}{*}{ CORE } & \multicolumn{5}{|c|}{$\begin{array}{l}\text { PI-92-AR-B3, water depth } 201 \mathrm{M} \\
\text { entire sample examined Species }\end{array}$} & & $\begin{array}{l}92-A R-B \\
1 / 128\end{array}$ & $\begin{array}{l}\text { 7, water } \\
\text { ample ex }\end{array}$ & $\begin{array}{l}\text { epth } 40 \\
\text { mined }\end{array}$ & $\begin{array}{l}M \\
\text { Species }\end{array}$ \\
\hline & $63-106$ & $106-125$ & $125-150$ & $>150$ & Total & 63-106 & $106-125$ & $125-150$ & $>150$ & total \\
\hline Adercotryma glomerata & 0 & 23 & 17 & 16 & 56 & 0 & $\overline{0}$ & 0 & 0 & 0 \\
\hline Alveophragmium crassimargo & 0 & 0 & 0 & 0 & 0 & 0 & 0 & 0 & 0 & 0 \\
\hline Ammodiscus catinus & 0 & 0 & 0 & 0 & 0 & 0 & 0 & 0 & 0 & 0 \\
\hline Ammotium cassis & 0 & 0 & 0 & 0 & 0 & 0 & 0 & 0 & 0 & 0 \\
\hline Astrononion gallowayi & 0 & 0 & 1 & 1 & 2 & 0 & 0 & 0 & 0 & 0 \\
\hline Bolivina arctica & 0 & 0 & 0 & 0 & 0 & 0 & 0 & 0 & 0 & 0 \\
\hline Bolivina pseudopunctata & 0 & 0 & 0 & 0 & 0 & 0 & 0 & 0 & 0 & 0 \\
\hline Buccella frigida & 21 & 10 & 6 & 1 & 38 & 0 & 0 & 0 & 0 & 0 \\
\hline Bulimina aculeata & 0 & 0 & 0 & 0 & 0 & 0 & 0 & 0 & 0 & 0 \\
\hline Cassidulina norcrossi & 0 & 8 & 83 & 127 & 218 & 0 & 0 & 0 & 5 & 5 \\
\hline Cassidulina teretis & 0 & 0 & 0 & 5 & 5 & 224 & 86 & 143 & 111 & 564 \\
\hline Chilostomella elongata & 0 & 0 & 0 & 0 & 0 & 0 & 0 & 0 & 0 & 0 \\
\hline Cibicides lobatulus & 0 & 0 & 0 & 0 & 0 & 9 & 3 & 1 & 9 & 22 \\
\hline Cribroelphidium clavatum & 14 & 0 & 4 & 0 & 18 & 0 & 0 & 0 & 0 & 0 \\
\hline Cribroelphidium excavatum & 33 & 28 & 33 & 23 & 117 & 0 & 0 & 0 & 0 & 0 \\
\hline Cribrostomoides sp. & 0 & 0 & 0 & 0 & 0 & 0 & 0 & 0 & 0 & 0 \\
\hline Cystammina argentea & 0 & 0 & 0 & 0 & 0 & 0 & 0 & 0 & 0 & 0 \\
\hline Dentalina pauperata & 0 & 0 & 0 & 0 & 0 & 0 & 0 & 0 & 0 & 0 \\
\hline Discorbis squamata & 6 & 0 & 0 & 0 & 6 & 0 & 0 & 0 & 0 & 0 \\
\hline Eggerella advena & 0 & 0 & 1 & 0 & 1 & 0 & 0 & 0 & 0 & 0 \\
\hline Eponides tumidulus & 0 & 0 & 0 & 0 & 0 & 0 & 0 & 0 & 0 & 0 \\
\hline Fontbotia wuellerstorfi & 0 & 0 & 0 & 0 & 0 & 0 & 0 & 0 & 0 & 0 \\
\hline Fursenkoina fusiformis & 29 & 13 & 5 & 1 & 48 & 0 & 0 & 0 & 0 & 0 \\
\hline Glandulina laevigata & 4 & 3 & 0 & 0 & 7 & 0 & 0 & 0 & 0 & 0 \\
\hline Globocassidulina subglobosa & 113 & 28 & 27 & 6 & 174 & 72 & 34 & 27 & 0 & 133 \\
\hline Glomospira gordialis & 0 & 0 & 0 & 0 & 0 & 0 & 0 & 0 & 0 & 0 \\
\hline Islandiella helenae & 52 & 48 & 0 & 0 & 100 & 0 & 0 & 0 & 0 & 0 \\
\hline Melonis barleeanum & 0 & 0 & 5 & 27 & 32 & 0 & 0 & 0 & 0 & 0 \\
\hline Nodosariids & 1 & 0 & 1 & 5 & 7 & 0 & 0 & 7 & 3 & 10 \\
\hline Nonionellina labradorica & 1 & 3 & 1 & 6 & 11 & 0 & 0 & 0 & 0 & 0 \\
\hline Oridorsalis tener & 0 & 0 & 0 & 0 & 0 & 0 & 1 & 1 & 1 & 3 \\
\hline Oridorsalis umbonatus & 0 & 0 & 0 & 0 & 0 & 0 & 0 & 0 & 0 & 0 \\
\hline Portatrochammina bipolaris & 0 & 4 & 0 & 0 & 4 & 0 & 1 & 0 & 0 & 1 \\
\hline Pullenia quinqueloba & 0 & 0 & 0 & 0 & 0 & 0 & 0 & 0 & 0 & 0 \\
\hline Quinqueloculina akneriana & 0 & 0 & 0 & 4 & 4 & 0 & 0 & 1 & 2 & 3 \\
\hline Quinqueloculina arctica & 0 & 0 & 0 & 0 & 0 & 0 & 0 & 0 & 0 & 0 \\
\hline Recurvoides scitulus & 0 & 0 & 0 & 0 & 0 & 0 & 0 & 0 & 0 & 0 \\
\hline Reophax scorpiurus & 0 & 0 & 0 & 0 & 0 & 0 & 0 & 0 & 0 & 0 \\
\hline Reophax spp. & 0 & 0 & 0 & 0 & 0 & 0 & 0 & 0 & 0 & 0 \\
\hline Robertinoides charlottensis & 0 & 0 & 1 & 0 & 1 & 1 & 0 & 0 & 0 & 1 \\
\hline Rosalina globularis & 0 & 0 & 0 & 0 & 0 & 0 & 0 & 0 & 0 & 0 \\
\hline Saccammina difflugiformis & 0 & 0 & 0 & 4 & 4 & 0 & 0 & 0 & 0 & 0 \\
\hline Spiroplectammina biformis & 4 & 0 & 0 & 0 & 4 & 0 & 0 & 0 & 0 & 0 \\
\hline Stainforthia concava & 1 & 3 & 0 & 1 & 5 & 15 & 4 & 1 & 0 & 20 \\
\hline Stetsonia horvathi & 148 & 2 & 0 & 0 & 150 & 66 & 0 & 0 & 0 & 66 \\
\hline Textularia kattagatensis & 0 & 1 & 0 & 0 & 1 & 0 & 0 & 0 & 0 & 0 \\
\hline Textularia torquata & 7 & 0 & 0 & 0 & 7 & 0 & 0 & 0 & 0 & 0 \\
\hline Triloculina frigida & 1 & 0 & 0 & 0 & 1 & 8 & 3 & 0 & 0 & 11 \\
\hline Trochammina pseudoinflata & 0 & 0 & 0 & 0 & 0 & 0 & 0 & 0 & 0 & 0 \\
\hline Trochammina subglobigeriniformis & 4 & 0 & 0 & 0 & 4 & 7 & 0 & 0 & 0 & 7 \\
\hline Valvulineria arctica & 0 & 1 & 0 & 0 & 1 & 1 & 2 & 0 & 0 & 3 \\
\hline Verneuilina minuta & 0 & 0 & 0 & 0 & 0 & 0 & 0 & 0 & 0 & 0 \\
\hline Size fraction total & 439 & $\overline{1775}$ & $\overline{185}$ & 2227 & 1026 & 403 & 134 & $\overline{181}$ & 131 & 849 \\
\hline Percent of Total Count & 42.79 & 17.06 & 18.03 & 22.12 & 100.0 & 47.47 & 15.78 & 21.32 & 15.43 & 100.0 \\
\hline S-WIndex & 0.85 & 0.79 & 0.70 & 0.54 & 1.01 & 0.51 & 0.42 & 0.31 & 0.28 & 0.48 \\
\hline
\end{tabular}




\begin{tabular}{|c|c|c|c|c|c|c|c|c|c|c|}
\hline \multirow[t]{2}{*}{ CORE } & \multicolumn{5}{|c|}{ PI-92-AR-B4, water depth $447 \mathrm{M}$} & & $\begin{array}{l}92-A R-B \\
\text { entire } s\end{array}$ & $\begin{array}{l}5, \text { water } \\
\text { ample ex }\end{array}$ & $\begin{array}{l}\text { pth } 558 \\
\text { mined }\end{array}$ & $\begin{array}{l}\text { M } \\
\text { Species }\end{array}$ \\
\hline & $63-106$ & $106-125$ & $125-150$ & $>150$ & total & 63-106 & $106-125$ & $125-150$ & $>150$ & total \\
\hline Adercotryma glomerata & 3 & 0 & 1 & $\overline{1}$ & $\overline{5}$ & 13 & 3 & 1 & $\overline{0}$ & 17 \\
\hline Alveophragmium crassimargo & 0 & 0 & 0 & 0 & 0 & 6 & 3 & 13 & 9 & 31 \\
\hline Ammodiscus catinus & 0 & 0 & 0 & 0 & 0 & 0 & 0 & 0 & 0 & 0 \\
\hline Ammotium cassis & 0 & 0 & 0 & 0 & 0 & 0 & 0 & 0 & 0 & 0 \\
\hline Astrononion gallowayi & 0 & 0 & 0 & 0 & 0 & 0 & 0 & 0 & 0 & 0 \\
\hline Bolivina arctica & 0 & 0 & 0 & 0 & 0 & 11 & 0 & 1 & 0 & 12 \\
\hline Bolivina pseudopunctata & 0 & 0 & 0 & 0 & 0 & 0 & 0 & 0 & 0 & 0 \\
\hline Buccella frigida & 0 & 0 & 0 & 0 & 0 & 2 & 0 & 0 & 0 & 2 \\
\hline Bulimina aculeata & 0 & 0 & 0 & 0 & 0 & 0 & 0 & 0 & 0 & 0 \\
\hline Cassidulina norcrossi & 0 & 0 & 0 & 0 & 0 & 21 & 0 & 0 & 1 & 22 \\
\hline Cassidulina teretis & 0 & 0 & 0 & 0 & 0 & 242 & 180 & 258 & 138 & 818 \\
\hline Chilostomella elongata & 0 & 0 & 0 & 0 & 0 & 0 & 0 & 0 & 0 & 0 \\
\hline Cibicides lobatulus & 0 & 0 & 0 & 0 & 0 & 0 & 0 & 0 & 0 & 0 \\
\hline Cribroelphidium clavatum & 0 & 0 & 0 & 0 & 0 & 0 & 1 & 0 & 0 & 1 \\
\hline Cribroelphidium excavatum & 0 & 0 & 0 & 0 & 0 & 0 & 3 & 0 & 1 & 4 \\
\hline Cribrostomoides sp. & 0 & 0 & 0 & 0 & 0 & 0 & 0 & 0 & 0 & 0 \\
\hline Cystammina argentea & 0 & 0 & 0 & 0 & 0 & 0 & 0 & 0 & 0 & 0 \\
\hline Dentalina pauperata & 0 & 0 & 0 & 0 & 0 & 0 & 0 & 0 & 1 & 1 \\
\hline Discorbis squamata & 0 & 0 & 0 & 0 & 0 & 1 & 0 & 0 & 0 & 1 \\
\hline Eggerella advena & 4 & 6 & 4 & 0 & 14 & 10 & 6 & 5 & 0 & 21 \\
\hline Eponides tumidulus & 0 & 0 & 0 & 0 & 0 & 0 & 0 & 0 & 0 & 0 \\
\hline Fontbotia wuellerstorfi & 0 & 0 & 0 & 0 & 0 & 0 & 0 & 0 & 0 & 0 \\
\hline Fursenkoina fusiformis & 0 & 0 & 0 & 0 & 0 & 0 & 1 & 0 & 0 & 1 \\
\hline Glandulina laevigata & 0 & 0 & 0 & 0 & 0 & 0 & 0 & 0 & 0 & 0 \\
\hline Globocassidulina subglobosa & 0 & 0 & 0 & 0 & 0 & 13 & 1 & 5 & 1 & 20 \\
\hline Glomospira gordialis & 1 & 0 & 0 & 0 & 1 & 37 & 7 & 6 & 1 & 51 \\
\hline Islandiella helenae & 0 & 0 & 0 & 0 & 0 & 0 & 0 & 0 & 3 & 3 \\
\hline Melonis barleeanum & 0 & 0 & 0 & 0 & 0 & 0 & 0 & 1 & 3 & 4 \\
\hline Nodosariids & 0 & 0 & 0 & 0 & 0 & 14 & 7 & 7 & 4 & 32 \\
\hline Nonionellina labradorica & 0 & 0 & 0 & 0 & 0 & 0 & 0 & 0 & 1 & 1 \\
\hline Oridorsalis tener & 0 & 0 & 0 & 0 & 0 & 0 & 0 & 3 & 12 & 15 \\
\hline Oridorsalis umbonatus & 0 & 0 & 0 & 0 & 0 & 0 & 0 & 0 & 0 & 0 \\
\hline Portatrochammina bipolaris & 33 & 13 & 16 & 0 & 62 & 29 & 20 & 15 & 2 & 66 \\
\hline Pullenia quinqueloba & 0 & 0 & 0 & 0 & 0 & 0 & 0 & 0 & 0 & 0 \\
\hline Quinqueloculina akneriana & 0 & 0 & 0 & 0 & 0 & 0 & 0 & 0 & 0 & 0 \\
\hline Quinqueloculina arctica & 0 & 0 & 0 & 0 & 0 & 0 & 0 & 3 & 5 & 8 \\
\hline Recurvoides scitulus & 0 & 0 & 0 & 1 & 1 & 0 & 0 & 0 & 166 & 166 \\
\hline Reophax scorpiurus & 0 & 0 & 0 & 0 & 0 & 0 & 0 & 3 & 0 & 3 \\
\hline Reophax spp. & 257 & 34 & 18 & 23 & 332 & 1198 & 117 & 39 & 78 & 1432 \\
\hline Robertinoides charlottensis & 0 & 0 & 0 & 0 & 0 & 0 & 0 & 0 & 0 & 0 \\
\hline Rosalina globularis & 0 & 0 & 0 & 0 & 0 & 0 & 0 & 0 & 0 & 0 \\
\hline Saccammina difflugiformis & 0 & 5 & 6 & 0 & 11 & 0 & 8 & 6 & 3 & 17 \\
\hline Spiroplectammina biformis & 5 & 1 & 0 & 0 & 6 & 0 & 5 & 4 & 0 & 9 \\
\hline Stainforthia concava & 0 & 0 & 0 & 0 & 0 & 10 & 3 & 0 & 0 & 13 \\
\hline Stetsonia horvathi & 0 & 0 & 0 & 0 & 0 & 22 & 0 & 0 & 0 & 22 \\
\hline Textularia kattagatensis & 0 & 0 & 0 & 0 & 0 & 4 & 0 & 0 & 0 & 4 \\
\hline Textularia torquata & 8 & 0 & 0 & 0 & 8 & 21 & 0 & 0 & 0 & 21 \\
\hline Triloculina frigida & 0 & 0 & 0 & 0 & 0 & 0 & 0 & 0 & 0 & 0 \\
\hline Trochammina pseudoinflata & 0 & 0 & 0 & 0 & 0 & 3 & 4 & 5 & 5 & 17 \\
\hline Trochammina subglobigeriniformis & 38 & 25 & 17 & 1 & 81 & 176 & 38 & 28 & 10 & 252 \\
\hline Valvulineria arctica & 0 & 0 & 0 & 0 & 0 & 7 & 2 & 0 & 0 & 9 \\
\hline Verneuilina minuta & 1 & 0 & 0 & 0 & 1 & 0 & 0 & 0 & 0 & 0 \\
\hline Size fraction total & 350 & 84 & 62 & 26 & 522 & 1840 & 409 & 403 & 444 & 3096 \\
\hline Percent of Total Count & 66.99 & 16.12 & 11.90 & 4.99 & 100.0 & 59.43 & 13.21 & 13.02 & 14.34 & 100.0 \\
\hline$S-W /$ Index & 0.29 & 0.46 & 0.48 & 0.10 & 0.37 & 0.70 & 0.57 & 0.40 & 0.58 & 0.70 \\
\hline
\end{tabular}




\begin{tabular}{|c|c|c|c|c|c|c|c|c|c|c|}
\hline \multirow[t]{3}{*}{ CORE } & \multicolumn{5}{|c|}{ PI-92-AR-B7, water depth $1,055 \mathrm{M}$} & \multicolumn{5}{|c|}{ PI-92-AR-B16, water depth $1,388 \mathrm{M}$} \\
\hline & \multirow[b]{2}{*}{ 63-106 } & \multicolumn{3}{|c|}{ 1/8 sample examined } & \multirow{2}{*}{$\begin{array}{c}\text { Species } \\
\text { total }\end{array}$} & \multirow[b]{2}{*}{ 63-106 } & \multicolumn{3}{|c|}{ 1/16 sample examined } & \multirow{2}{*}{$\begin{array}{c}\text { Species } \\
\text { total }\end{array}$} \\
\hline & & $106-125$ & $125-150$ & $>150$ & & & $106-125$ & $125-150$ & $>150$ & \\
\hline Adercotryma glomerata & 0 & 0 & 0 & 0 & 0 & 0 & $\overline{0}$ & 0 & 0 & 0 \\
\hline Alveophragmium crassimargo & 0 & 0 & 0 & 0 & 0 & 0 & 0 & 0 & 0 & 0 \\
\hline Ammodiscus catinus & 0 & 0 & 0 & 0 & 0 & 0 & 0 & 0 & 0 & 0 \\
\hline Ammotium cassis & 0 & 0 & 0 & 0 & 0 & 0 & 0 & 0 & 0 & 0 \\
\hline Astrononion gallowayi & 0 & 0 & 0 & 0 & 0 & 0 & 0 & 0 & 0 & 0 \\
\hline Bolivina arctica & 1 & 0 & 0 & 0 & 1 & 6 & 0 & 0 & 0 & 6 \\
\hline Bolivina pseudopunctata & 1 & 0 & 0 & 0 & 1 & 0 & 0 & 0 & 0 & 0 \\
\hline Buccella frigida & 0 & 0 & 0 & 0 & 0 & 0 & 0 & 0 & 0 & 0 \\
\hline Bulimina aculeata & 0 & 0 & 0 & 0 & 0 & 0 & 0 & 0 & 0 & 0 \\
\hline Cassidulina norcrossi & 0 & 0 & 0 & 0 & 0 & 1 & 0 & 0 & 1 & 2 \\
\hline Cassidulina teretis & 43 & 39 & 54 & 53 & 189 & 19 & 6 & 2 & 13 & 40 \\
\hline Chilostomella elongata & 0 & 0 & 0 & 0 & 0 & 0 & 0 & 0 & 0 & 0 \\
\hline Cibicides lobatulus & 0 & 0 & 0 & 2 & 2 & 2 & 0 & 1 & 0 & 3 \\
\hline Cribroelphidium clavatum & 0 & 0 & 0 & 0 & 0 & 0 & 0 & 0 & 0 & 0 \\
\hline Cribroeiphidium excavatum & 0 & 1 & 0 & 0 & 1 & 0 & 1 & 0 & 0 & 1 \\
\hline Cribrostomoides sp. & 0 & 0 & 0 & 4 & 4 & 0 & 0 & 0 & 0 & 0 \\
\hline Cystammina argentea & 0 & 0 & 0 & 0 & 0 & 0 & 0 & 0 & 0 & 0 \\
\hline Dentalina pauperata & 0 & 0 & 0 & 1 & 1 & 0 & 0 & 0 & 0 & 0 \\
\hline Discorbis squamata & 0 & 0 & 0 & 0 & 0 & 0 & 0 & 0 & 0 & 0 \\
\hline Eggerella advena & 0 & 0 & 0 & 0 & 0 & 0 & 0 & 0 & 0 & 0 \\
\hline Eponides tumidulus & 129 & 78 & 12 & 0 & 219 & 259 & 53 & 6 & 0 & 318 \\
\hline Fontbotia wuellerstorfi & 0 & 0 & 0 & 15 & 15 & 1 & 0 & 0 & 17 & 18 \\
\hline Fursenkoina fusiformis & 0 & 0 & 0 & 0 & 0 & 6 & 0 & 0 & 0 & 6 \\
\hline Glandulina laevigata & 0 & 0 & 0 & 0 & 0 & 0 & 0 & 0 & 0 & 0 \\
\hline Globocassidulina subglobosa & 0 & 0 & 0 & 0 & 0 & 0 & 0 & 0 & 0 & 0 \\
\hline Glomospira gordialis & 0 & 0 & 0 & 0 & 0 & 7 & 0 & 2 & 0 & 9 \\
\hline Islandiella helenae & 0 & 0 & 0 & 0 & 0 & 0 & 0 & 0 & 0 & 0 \\
\hline Melonis barleeanum & 0 & 0 & 0 & 0 & 0 & 0 & 0 & 0 & 0 & 0 \\
\hline Nodosariids & 8 & 2 & 3 & 1 & 14 & 9 & 0 & 5 & 0 & 14 \\
\hline Nonionellina labradorica & 0 & 0 & 0 & 0 & 0 & 0 & 0 & 0 & 0 & 0 \\
\hline Oridorsalis tener & 16 & 25 & 50 & 69 & 160 & 89 & 30 & 83 & 61 & 263 \\
\hline Oridorsalls umbonatus & 0 & 0 & 0 & 0 & 0 & 0 & 0 & 0 & 0 & 0 \\
\hline Portatrochammina bipolaris & 0 & 0 & 0 & 0 & 0 & 0 & 0 & 0 & 0 & 0 \\
\hline Pullenia quinqueloba & 0 & 0 & 0 & 0 & 0 & 0 & 0 & 0 & 0 & 0 \\
\hline Quinqueloculina akneriana & 0 & 0 & 0 & 0 & 0 & 1 & 0 & 9 & 24 & 34 \\
\hline Quinqueloculina arctica & 0 & 0 & 0 & 72 & 72 & 0 & 0 & 0 & 0 & 0 \\
\hline Recurvoides scitulus & 0 & 0 & 0 & 0 & 0 & 0 & 0 & 0 & 0 & 0 \\
\hline Reophax scorpiurus & 0 & 0 & 0 & 0 & 0 & 0 & 0 & 0 & 0 & 0 \\
\hline Reophax spp. & 0 & 0 & 0 & 0 & 0 & 4 & 0 & 0 & 0 & 4 \\
\hline Robertinoides charlottensis & 1 & 0 & 0 & 0 & 1 & 1 & 3 & 2 & 0 & 6 \\
\hline Rosalina globularis & 0 & 0 & 0 & 0 & 0 & 0 & 0 & 0 & 0 & 0 \\
\hline Saccammina difflugiformis & 0 & 0 & 0 & 0 & 0 & 0 & 0 & 0 & 0 & 0 \\
\hline Spiroplectammina biformis & 0 & 0 & 0 & 0 & 0 & 0 & 0 & 0 & 0 & 0 \\
\hline Stainforthia concava & 10 & 0 & 0 & 0 & 10 & 0 & 0 & 0 & 0 & 0 \\
\hline Stetsonia horvathi & 96 & 0 & 0 & 0 & 96 & 163 & 1 & 0 & 0 & 164 \\
\hline Textularia kattagatensis & 0 & 0 & 0 & 0 & 0 & 0 & 0 & 0 & 0 & 0 \\
\hline Textularia torquata & 0 & 0 & 0 & 0 & 0 & 0 & 0 & 0 & 0 & 0 \\
\hline Triloculina frigida & 18 & 15 & 16 & 4 & 53 & 12 & 1 & 15 & 0 & 28 \\
\hline Trochammina pseudoinflata & 0 & 0 & 0 & 0 & 0 & 1 & 3 & 0 & 0 & 4 \\
\hline Trochammina subglobigeriniformis & 0 & 0 & 0 & 0 & 0 & 0 & 0 & 0 & 0 & 0 \\
\hline Valvulineria arctica & 67 & 9 & 7 & 0 & 83 & 24 & 17 & 6 & 1 & 48 \\
\hline Verneullina minuta & 0 & 0 & 0 & 0 & 0 & 1 & 0 & 0 & 0 & 1 \\
\hline Size fraction total & 390 & 169 & 142 & 221 & 922 & 606 & 115 & 131 & 117 & 969 \\
\hline Percent of Total Count & 42.30 & 18.33 & 15.40 & 23.97 & 100.0 & 62.50 & 11.88 & 13.53 & 12.09 & 100.0 \\
\hline S-W Index & 0.76 & 0.62 & 0.62 & 0.65 & 0.89 & 0.67 & 0.57 & 0.48 & 0.55 & 0.76 \\
\hline
\end{tabular}




\begin{tabular}{|c|c|c|c|c|c|c|c|c|c|c|}
\hline \multirow[t]{2}{*}{ CORE } & \multicolumn{5}{|c|}{ PI-92-AR-B8, water depth $1,402 \mathrm{M}$} & \multicolumn{5}{|c|}{ PI-92-AR-B9, water depth 2,120 M } \\
\hline & 63-106 & $106-125$ & $125-150$ & $>150$ & total & $63-106$ & $106-125$ & $125-150$ & $>150$ & total \\
\hline Adercotryma glomerata & 0 & 0 & 0 & 0 & 0 & 1 & 0 & 0 & 0 & 1 \\
\hline Alveophragmium crassimargo & 0 & 0 & 0 & 0 & 0 & 0 & 1 & 0 & 0 & 1 \\
\hline Ammodiscus catinus & 0 & 0 & 0 & 0 & 0 & 0 & 0 & 0 & 0 & 0 \\
\hline Ammotium cassis & 0 & 0 & 0 & 0 & 0 & 0 & 0 & 0 & 0 & 0 \\
\hline Astrononion gallowayi & 0 & 0 & 0 & 0 & 0 & 0 & 0 & 0 & 0 & 0 \\
\hline Bolivina arctica & 3 & 0 & 0 & 0 & 3 & 11 & 0 & 0 & 0 & 11 \\
\hline Bolivina pseudopunctata & 0 & 0 & 0 & 0 & 0 & 0 & 0 & 0 & 0 & 0 \\
\hline Buccella frigida & 0 & 1 & 2 & 2 & 5 & 0 & 0 & 0 & 0 & 0 \\
\hline Bulimina aculeata & 0 & 0 & 0 & 0 & 0 & 0 & 0 & 0 & 0 & 0 \\
\hline Cassidulina norcrossi & 0 & 0 & 0 & 0 & 0 & 0 & 0 & 0 & 0 & 0 \\
\hline Cassidulina teretis & 17 & 17 & 10 & 13 & 57 & 0 & 0 & 0 & 0 & 0 \\
\hline Chilostomella elongata & 1 & 0 & 0 & 0 & 1 & 0 & 0 & 0 & 0 & 0 \\
\hline Cibicides lobatulus & 0 & 0 & 0 & 0 & 0 & 0 & 0 & 0 & 0 & 0 \\
\hline Cribroelphidium clavatum & 0 & 0 & 0 & 0 & 0 & 0 & 0 & 0 & 0 & 0 \\
\hline Cribroelphidium excavatum & 0 & 0 & 0 & 0 & 0 & 0 & 0 & 0 & 0 & 0 \\
\hline Cribrostomoides sp. & 0 & 0 & 0 & 0 & 0 & 0 & 0 & 0 & 0 & 0 \\
\hline Cystammina argentea & 0 & 0 & 0 & 0 & 0 & 4 & 0 & 0 & 0 & 4 \\
\hline Dentalina pauperata & 0 & 0 & 0 & 0 & 0 & 0 & 0 & 0 & 0 & 0 \\
\hline Discorbis squamata & 0 & 0 & 0 & 0 & 0 & 0 & 0 & 0 & 0 & 0 \\
\hline Eggerella advena & 0 & 0 & 0 & 0 & 0 & 0 & 0 & 0 & 0 & 0 \\
\hline Eponides tumidulus & 265 & 57 & 12 & 0 & 334 & 11 & 34 & 6 & 0 & 51 \\
\hline Fontbotia wuellerstorfi & 14 & 0 & 3 & 19 & 36 & 0 & 4 & 4 & 28 & 36 \\
\hline Fursenkoina fusiformis & 1 & 0 & 0 & 0 & 1 & 0 & 0 & 0 & 0 & 0 \\
\hline Glandulina laevigata & 0 & 0 & 0 & 0 & 0 & 0 & 0 & 0 & 0 & 0 \\
\hline Globocassidulina subglobosa & 2 & 0 & 0 & 0 & 2 & 0 & 0 & 1 & 0 & 1 \\
\hline Glomospira gordialis & 3 & 0 & 0 & 0 & 3 & 1 & 1 & 1 & 0 & 3 \\
\hline Islandiella helenae & 0 & 0 & 0 & 0 & 0 & 0 & 0 & 0 & 0 & 0 \\
\hline Melonis barleeanum & 0 & 3 & 0 & 0 & 3 & 0 & 0 & 0 & 0 & 0 \\
\hline Nodosariìds & 7 & 7 & 2 & 0 & 16 & 24 & 3 & 1 & 1 & 29 \\
\hline Nonionellina labradorica & 0 & 0 & 0 & 0 & 0 & 0 & 0 & 0 & 0 & 0 \\
\hline Oridorsalis tener & 92 & 50 & 124 & 116 & 382 & 207 & 102 & 203 & 174 & 686 \\
\hline Oridorsalis umbonatus & 0 & 0 & 0 & 1 & 1 & 0 & 0 & 0 & 0 & 0 \\
\hline Portatrochammina bipolaris & 0 & 0 & 0 & 0 & 0 & 0 & 0 & 0 & 0 & 0 \\
\hline Pullenia quinqueloba & 0 & 1 & 0 & 0 & 1 & 0 & 0 & 0 & 0 & 0 \\
\hline Quinqueloculina akneriana & 0 & 0 & 0 & 8 & 8 & 0 & 5 & 3 & 2 & 10 \\
\hline Quinqueloculina arctica & 0 & 0 & 0 & 0 & 0 & 0 & 0 & 0 & 10 & 10 \\
\hline Recurvoides scitulus & 0 & 0 & 0 & 0 & 0 & 0 & 0 & 0 & 1 & 1 \\
\hline Reophax scorpiurus & 0 & 0 & 0 & 0 & 0 & 0 & 0 & 0 & 0 & 0 \\
\hline Reophax spp. & 0 & 0 & 2 & 0 & 2 & 2 & 1 & 0 & 0 & 3 \\
\hline Robertinoides charlottensis & 0 & 0 & 1 & 1 & 2 & 3 & 1 & 0 & 1 & 5 \\
\hline Rosalina globularis & 0 & 0 & 0 & 0 & 0 & 0 & 0 & 0 & 0 & 0 \\
\hline Saccammina difflugiformis & 0 & 0 & 0 & 0 & 0 & 0 & 0 & 0 & 0 & 0 \\
\hline Spiroplectammina biformis & 0 & 0 & 0 & 0 & 0 & 0 & 0 & 0 & 0 & 0 \\
\hline Stainforthia concava & 6 & 0 & 0 & 0 & 6 & 4 & 0 & 0 & 0 & 4 \\
\hline Stetsonia horvathi & 475 & 0 & 1 & 0 & 476 & 1544 & 5 & 0 & 0 & 1549 \\
\hline Textularia kattagatensis & 0 & 0 & 0 & 0 & 0 & 0 & 0 & 0 & 0 & 0 \\
\hline Textularia torquata & 0 & 0 & 0 & 0 & 0 & 0 & 0 & 0 & 0 & 0 \\
\hline Triloculina frigida & 45 & 19 & 11 & 1 & 76 & 129 & 25 & 27 & 9 & 190 \\
\hline Trochammina pseudoinflata & 0 & 0 & 0 & 0 & 0 & 0 & 0 & 0 & 0 & 0 \\
\hline Trochammina subglobigeriniformis & 0 & 0 & 0 & 0 & 0 & 6 & 1 & 0 & 0 & 7 \\
\hline Valvulineria arctica & 40 & 4 & 5 & 0 & 49 & 75 & 0 & 0 & 0 & 75 \\
\hline Verneuilina minuta & 0 & 0 & 0 & 0 & 0 & 0 & 0 & 0 & 0 & 0 \\
\hline Size fraction total & 971 & 159 & 173 & 161 & 1464 & 2022 & 183 & 246 & 226 & 2677 \\
\hline Percent of Total Count & 66.33 & 10.86 & 11.82 & 11.00 & 100.0 & 75.53 & 6.84 & 9.19 & 8.44 & 100.0 \\
\hline S-WIndex & 0.60 & 0.69 & 0.48 & 0.43 & 0.75 & 0.50 & 0.60 & 0.31 & 0.36 & 0.62 \\
\hline
\end{tabular}




\begin{tabular}{|c|c|c|c|c|c|c|c|c|c|c|}
\hline \multirow[t]{3}{*}{ CORE } & \multicolumn{5}{|c|}{ PI-92-AR-B15, water depth 2,135 M } & \multicolumn{5}{|c|}{ PI-92-AR-B12, water depth 3,811 M } \\
\hline & & $1 / 8 \mathrm{~s}$ & mple exa & ined & Species & & entires & ample ex & $\min \theta d$ & Species \\
\hline & 63-106 & $106-125$ & $125-150$ & $>150$ & total & 63-106 & $106-125$ & $125-150$ & $>150$ & total \\
\hline Adercotryma glomerata & 7 & 1 & 0 & 0 & 8 & 2 & 0 & 0 & 0 & 2 \\
\hline Alveophragmium crassimargo & 0 & 0 & 0 & 0 & 0 & 3 & 0 & 0 & 0 & 3 \\
\hline Ammodiscus catinus & 0 & 0 & 1 & 0 & 1 & 1 & 0 & 0 & 0 & 1 \\
\hline Ammotium cassis & 0 & 0 & 0 & 0 & 0 & 0 & 0 & 0 & 0 & 0 \\
\hline Astrononion gallowayi & 0 & 0 & 0 & 0 & 0 & 0 & 0 & 0 & 0 & 0 \\
\hline Bolivina arctica & 0 & 0 & 0 & 0 & 0 & 2 & 0 & 0 & 0 & 2 \\
\hline Bolivina pseudopunctata & 0 & 0 & 0 & 0 & 0 & 0 & 0 & 0 & 0 & 0 \\
\hline Buccella frigida & 0 & 0 & 0 & 0 & 0 & 0 & 0 & 1 & 0 & 1 \\
\hline Bulimina aculeata & 0 & 0 & 0 & 0 & 0 & 0 & 0 & 0 & 0 & 0 \\
\hline Cassidulina norcrossi & 0 & 0 & 0 & 0 & 0 & 0 & 0 & 0 & 0 & 0 \\
\hline Cassidulina teretis & 0 & 0 & 0 & 0 & 0 & 0 & 0 & 0 & 0 & 0 \\
\hline Chilostomella elongata & 1 & 0 & 0 & 0 & 1 & 0 & 0 & 0 & 0 & 0 \\
\hline Cibicides lobatulus & 54 & 7 & 0 & 0 & 61 & 1 & 1 & 0 & 0 & 2 \\
\hline Cribroelphidium clavatum & 0 & 0 & 0 & 0 & 0 & 2 & 0 & 0 & 1 & 3 \\
\hline Cribroelphidium excavatum & 0 & 0 & 0 & 0 & 0 & 2 & 0 & 0 & 0 & 2 \\
\hline Cribrostomoides sp. & 0 & 0 & 0 & 0 & 0 & 0 & 0 & 0 & 0 & 0 \\
\hline Cystammina argentea & 0 & 0 & 0 & 0 & 0 & 0 & 0 & 0 & 0 & 0 \\
\hline Dentalina pauperata & 0 & 0 & 0 & 0 & 0 & 0 & 0 & 0 & 0 & 0 \\
\hline Discorbis squamata & 0 & 0 & 0 & 0 & 0 & 1 & 0 & 0 & 0 & 1 \\
\hline Eggerella advena & 1 & 0 & 0 & 0 & 1 & 0 & 1 & 0 & 0 & 1 \\
\hline Eponides tumidulus & 225 & 20 & 9 & 0 & 254 & 38 & 16 & 7 & 1 & 62 \\
\hline Fontbotia wuellerstorfi & 0 & 0 & 20 & 115 & 135 & 0 & 0 & 0 & 0 & 0 \\
\hline Fursenkoina fusiformis & 2 & 0 & 0 & 0 & 2 & 0 & 0 & 0 & 0 & 0 \\
\hline Glandulina laevigata & 0 & 0 & 0 & 0 & 0 & 0 & 0 & 0 & 0 & 0 \\
\hline Globocassidulina subglobosa & 0 & 0 & 0 & 0 & 0 & 1 & 0 & 0 & 0 & 1 \\
\hline Glomospira gordialis & 12 & 2 & 0 & 0 & 14 & 2 & 0 & 0 & 0 & 2 \\
\hline Islandiella helenae & 0 & 0 & 0 & 0 & 0 & 0 & 0 & 0 & 0 & 0 \\
\hline Melonis barleeanum & 0 & 0 & 0 & 0 & 0 & 0 & 0 & 0 & 0 & 0 \\
\hline Nodosariids & 13 & 3 & 4 & 3 & 23 & 0 & 0 & 3 & 5 & 8 \\
\hline Nonionellina labradorica & 0 & 0 & 0 & 0 & 0 & 0 & 0 & 0 & 0 & 0 \\
\hline Oridorsalis tener & 73 & 40 & 80 & 71 & 264 & 14 & 18 & 35 & 20 & 87 \\
\hline Oridorsalis umbonatus & 0 & 0 & 0 & 0 & 0 & 0 & 0 & 0 & 0 & 0 \\
\hline Portatrochammina bipolaris & 13 & 0 & 0 & 0 & 13 & 1 & 0 & 0 & 0 & 1 \\
\hline Pullenia quinqueloba & 0 & 0 & 0 & 0 & 0 & 0 & 0 & 0 & 0 & 0 \\
\hline Quinqueloculina akneriana & 14 & 5 & 6 & 23 & 48 & 0 & 0 & 0 & 0 & 0 \\
\hline Quinqueloculina arctica & 0 & 0 & 0 & 0 & 0 & 0 & 0 & 0 & 0 & 0 \\
\hline Recurvoides scitulus & 0 & 0 & 0 & 0 & 0 & 0 & 0 & 0 & 0 & 0 \\
\hline Reophax scorpiurus & 0 & 0 & 0 & 0 & 0 & 0 & 0 & 0 & 0 & 0 \\
\hline Reophax spp. & 33 & 0 & 0 & 0 & 33 & 0 & 0 & 0 & 0 & 0 \\
\hline Robertinoides charlottensis & 0 & 0 & 4 & 0 & 4 & 0 & 0 & 0 & 0 & 0 \\
\hline Rosalina globularis & 0 & 0 & 0 & 0 & 0 & 0 & 0 & 0 & 0 & 0 \\
\hline Saccammina difflugiformis & 2 & 0 & 0 & 0 & 2 & 0 & 0 & 0 & 0 & 0 \\
\hline Spiroplectammina biformis & 0 & 0 & 0 & 0 & 0 & 0 & 0 & 1 & 0 & 1 \\
\hline Stainforthia concava & 4 & 0 & 0 & 0 & 4 & 0 & 0 & 0 & 0 & 0 \\
\hline Stetsonia horvathi & 131 & 0 & 0 & 0 & $13 t$ & 74 & 0 & 0 & 0 & 74 \\
\hline Textularia kattagatensis & 0 & 0 & 0 & 0 & 0 & 0 & 0 & 0 & 0 & 0 \\
\hline Textularia torquata & 0 & 0 & 0 & 0 & 0 & 0 & 0 & 0 & 0 & 0 \\
\hline Triloculina frigida & 40 & 4 & 6 & 0 & 50 & 0 & 1 & 0 & 1 & 2 \\
\hline Trochammina pseudoinflata & 1 & 1 & 0 & 0 & 2 & 0 & 0 & 0 & 0 & 0 \\
\hline Trochammina subglobigeriniformis & 2 & 0 & 0 & 0 & 2 & 5 & 0 & 0 & 0 & 5 \\
\hline Valvulineria arctica & 118 & 0 & 0 & 0 & 118 & 0 & 0 & 0 & 0 & 0 \\
\hline Verneuilina minuta & 6 & 0 & 0 & 0 & 6 & 0 & 0 & 0 & 0 & 0 \\
\hline Size fraction total & 752 & 83 & 130 & 212 & 1177 & 149 & 37 & 47 & 28 & 261 \\
\hline Percent of Total Count & 63.68 & 7.09 & 11.11 & 18.12 & 100.00 & 57.09 & 14.18 & 18.01 & 10.73 & 100.0 \\
\hline S-WIndex & 0.81 & 0.56 & 0.51 & 0.43 & 0.88 & 0.57 & 0.35 & 0.21 & 0.06 & 0.66 \\
\hline
\end{tabular}

